\title{
Set and Setting: A Randomized Study of Different Musical Genres in Supporting
}

\section{Psychedelic Therapy}

Justin C. Strickland ${ }^{1}$, Albert Garcia-Romeu ${ }^{1}$, and Matthew W. Johnson ${ }^{1 *}$

${ }^{1}$ Department of Psychiatry and Behavioral Sciences, Johns Hopkins University School of Medicine, 5510 Nathan Shock Drive, Baltimore, MD 21224, USA

${ }^{*}$ Correspondence concerning this article should be addressed to Matthew W. Johnson, Department of Psychiatry and Behavioral Sciences, Johns Hopkins University School of Medicine, 5510 Nathan Shock Drive, Baltimore, MD 21224-6823, USA. Email: mwj@jhu.edu

This manuscript was accepted for publication on December 11, 2020 by ACS Pharmacology \& Translational Science. This is not the copy of record. 


\begin{abstract}
Mounting evidence supports the serotonin $2 \mathrm{~A}$ receptor agonist psilocybin as a psychiatric pharmacotherapy. Little research has experimentally examined how session "set and setting" impacts subjective and therapeutic effects. We analyzed effects of musical genre played during sessions of a psilocybin study for tobacco smoking cessation. Participants $(N=10)$ received psilocybin $(20-30 \mathrm{mg} / 70 \mathrm{~kg})$ in two sessions, each with a different genre (Western classical versus overtone-based), with order counterbalanced. Participants chose one genre for a third session (30mg/70kg). Mystical experiences scores tended to be higher in overtone-based than Western classical sessions. Six of ten participants chose overtone-based music for a third session. Biologically-confirmed smoking abstinence was similar based on musical choice, with a slight benefit for participants choosing the overtone-based playlist (66.7\% versus $50 \%)$. These data call into question whether Western classical music typically used in psychedelic therapy holds unique benefit. Broadly, they call for experimentally examining session components toward optimizing psychedelic therapeutic protocols.
\end{abstract}

Keywords: Cessation; Cigarettes; Music; Psilocybin; Psychedelic; Nicotine 
The past decade has witnessed a resurgent interest in the therapeutic potential of classic psychedelic drugs in both the scientific and popular press. ${ }^{1-3}$ Classic psychedelics (i.e., psilocin, lysergic acid diethylamide [LSD], and dimethyltryptamine [DMT]) act as agonists at the serotonin $2 \mathrm{~A}$ receptor $\left(5-\mathrm{HT}_{2 \mathrm{~A}} \mathrm{R}\right)$ - the mechanism by which positive clinical effects are thought to be mediated. ${ }^{2}$ Contemporary clinical trials with psilocybin (i.e., the prodrug for psilocin present along with psilocin in many species of mushrooms), LSD, and ayahuasca (which contains DMT) have reported efficacy for varied psychiatric outcomes to include depression and anxiety related to cancer and other life-threatening illness, treatment-resistant depression, tobacco use disorder, and alcohol use disorder. ${ }^{1}$ Notably, these findings correspond with those from studies conducted some 50 years prior with LSD which also reported promising findings for affective, anxiety, and substance use disorder symptoms. ${ }^{1}$

Psychedelic science has long recognized the centrality of set (i.e., psychological state of a person) and setting (i.e., a person's environmental context) when determining the behavioral and clinical effects of $5-\mathrm{HT}_{2 \mathrm{~A}} \mathrm{R}$ compounds. ${ }^{4-6}$ Traditional laboratory contexts that contain overtly "sterile" stimuli (e.g., white walls, medical equipment), for instance, have been suggested to increase the likelihood of negative reactions to psychedelic drugs. ${ }^{7}$ This relevance of set and setting contributed, in part, to the development of a standardized "psychedelic therapy" for use with 5- $\mathrm{HT}_{2 \mathrm{~A}} \mathrm{R}$ agonists; a method that has remained largely unchanged since its inception in the 1950s and 60s. ${ }^{8}$ These approaches involve highly specific recommendations for psychedelic administration and patient experience, yet very little research has systematically evaluated how variations in the delivery and deviations within or from this standardization may impact subjective and therapeutic effects.

Early observations suggested environmental and psychological factors such as the number of people present during drug administration sessions, and feeling socially supported or not throughout the drug administration process could have an important influence on psilocybin effects. ${ }^{9}$ One experimental study randomized 176 men in inpatient alcoholism treatment to receive 
either 1) no drug or therapy, 2) LSD alone with no therapy during sessions, 3) LSD with active talk psychotherapy during sessions, or 4) LSD with hypnosis based therapy during sessions, finding no substantial between group differences in long-term outcomes. ${ }^{10}$ However, this was conceptualized as an experimental manipulation of the presence or type of therapy rather than of setting.

Another study randomized 75 healthy individuals to receive either 1) very low dose psilocybin with moderate duration of preparation and post-session support, 2) a high dose of psilocybin with moderate duration of preparation and post-session support, or 3) a high dose of psilocybin with a higher duration of preparation and additional post-session support, finding that both greater dose and support were associated with sustained improvements in wellbeing. ${ }^{11}$ However, while different levels of psilocybin session preparation may be considered a manipulation of set and setting, ${ }^{5}$ the sessions themselves were conducted in an identical fashion. Moreover, the groups differed by not only preparation duration, but also the extent and type of post-session support, confounding any effect of preparation on outcomes.

Specifically relevant to the current analysis, one study evaluated five different musical contexts (e.g., no music, familiar music, unfamiliar music) presented during LSD sessions for alcohol use. ${ }^{12}$ That study found no significant differences in participant reported effects of music or observer-rated effects of LSD, although the between-subject design, quasi-randomization (i.e., the first two cohorts of participants were not randomly assigned), and absence of an objective clinical outcome limits interpretation of those findings. A goal of the present analysis was to directly and experimentally manipulate, by random assignment, session set and setting factors in a contemporary clinical context to address these limitations.

Music is one standard feature in clinical psychedelic therapy as well as a consistent facet of the broader cultural and research context surrounding psychedelic drugs. ${ }^{13}$ Music and $5-\mathrm{HT}_{2 \mathrm{~A}} \mathrm{R}$ agonist drugs mechanistically relate in several neurobiological and neurochemical ways. For example, 5- $\mathrm{HT}_{2 \mathrm{~A}} \mathrm{R}$ signaling modulates neural response to music and other auditory responses 
across a cochlear-to-auditory cortex pathway. ${ }^{14,}{ }^{15}$ Psychedelic drugs, specifically, have been shown to alter the neural response to the acoustic properties of music with a role for $5-\mathrm{HT}_{2 \mathrm{~A}}$ signaling. ${ }^{16}$ Clinical guidelines have often emphasized the use of music during psychedelic therapy with specific recommendations about how music sequencing should match the structure of a psychedelic drug's time course. ${ }^{4,6,17}$ In fact, use of music in psychedelic therapy is not unique to contemporary settings - historical records emphasize the prominence of music in ancient medicinal and ceremonial contexts involving psychedelics. ${ }^{13}$

Psychedelic therapy has almost exclusively used classical music, predominately Western classical pieces, likely due to recommendations present in early guidelines. For instance, one of the most commonly cited works on music and music sequencing in psychedelic therapy noted "Our most effective music was classical, light classical at the beginning (as well as Hindu ragas), and especially concertos during the body of the session" (p. 215). ${ }^{4}$ Similarly, modern safety guidelines for psychedelic research describe psychedelic therapy as "supportive interpersonal conditions, with the patient wearing eyeshades and listening to classical music through headphones during the course of pharmacological action" (p. 605; emphasis added). ${ }^{6}$ Nonetheless, variations in musical structures and individual differences in preferred acoustic properties could impact therapeutic outcomes. Existing evidence supports this hypothesis with one study finding that greater liking, resonance, and openness toward standardized music played in psychedelic sessions was associated with larger reductions in depressive symptoms a week later. ${ }^{18}$

The purpose of the current analysis was to experimentally evaluate the effects of musical genre - Western classical (as typically used in psychedelic studies) versus overtone-heavy instruments - on session experience and smoking cessation outcomes among nicotinedependent smokers participating in an open-label study. ${ }^{20,21}$ Participants completed therapeutic psilocybin sessions with the two different musical playlists accompanying those sessions. One (hereafter referred to as the "Western classical" playlist) was largely based on Western classical 
music and largely resembles playlists used in previous psilocybin studies by our group and others. ${ }^{21}$ The other playlist (hereafter referred to as the "overtone-based playlist") emphasized instruments with a particularly strong overtone signature (e.g. Tibetan singing bowls, gongs, digeridoo, chimes, bells, sitar, human voice overtone singing). Some of the overtone-based playlist tracks lacked traditionally identifiable melody and/or rhythm. The playlist used was counterbalanced across two sessions with study outcomes analyzed by music genre. Although conducted with a small sample, this analysis utilized a randomized within-subject design, and as such constitutes the first study to our knowledge of an experimental comparison of session set and setting reported in a half century, and first fully randomized within-subject study of session set and setting conditions evaluating psychedelic experiences and objective therapeutic outcomes. Moreover, the experimental rigor of the study was enhanced by the inclusion of a choice procedure in which, after sampling each of two musical playlists across the first two psilocybin sessions, participants chose their preferred playlist for a third session. This and future such studies will contribute to the clinical application of $5-\mathrm{HT}_{2 \mathrm{~A}} \mathrm{R}$ agonists in psychiatric health by identifying the core features of psychedelic therapy as well as those that may be modified, removed, or individualized to optimize patient-specific therapeutic outcomes.

\section{Results}

\section{Mystical and Challenging Experiences}

On session days after the resolution of drug effects, participants completed standardized subjective effect measures evaluating mystical experiences (e.g., feelings of unity, transcendence of space/time) $)^{24}$ and challenging experiences (e.g., panic, feelings of losing sanity) ${ }^{26}$ that occurred during the psilocybin session. Figure 1 contains mystical experience questionnaire (MEQ30) scores for individual participant divided by session and dose received $(20 \mathrm{mg} / 70 \mathrm{~kg}$ or $30 \mathrm{mg} / 70$ $\mathrm{kg}$ ). Visual inspection of individual and average data indicated higher overall scores for overtonebased sessions (mean=0.68; $S D=0.20$ ) compared to Western classical sessions (mean=0.52; $S D=0.21)$. This difference was of a medium effect size (Cohen's $d_{z}=0.57$ ), but was not statistically 
significant, $p=.107$. Controlling for psilocybin dose in a random intercept mixed effect model did not attenuate the magnitude of the difference by musical genre. Specifically, although these effects did not reach a threshold of statistical significance, higher MEQ30 scores were observed for overtone-based sessions, $b=0.14, p=.108$, and higher MEQ30 scores were observed in the 30 $\mathrm{mg} / 70 \mathrm{~kg}$ sessions, $b=0.14, p=.102$.

Figure 2 contains Challenging Experience Questionnaire (CEQ) scores for individual participants by session and dose. Visual inspection of individual and average data indicated no apparent pattern or differences between overtone-based sessions (mean=0.33; $S D=0.20$ ) and Western classical sessions (mean=0.34; $\mathrm{SD}=0.22$ ). Comparisons by musical genre were not statistically significant, $p=.74$, and of less than a small effect size, $d_{z}=-0.11$.

\section{Musical Genre Selection}

Participants were provided the opportunity to select their preferred musical playlist for the completion of a third psilocybin session with the high dose (30 mg/70 kg). Selections were roughly equally distributed with $60 \%(6 / 10)$ of participants selecting the overtone-based playlist. These selections were not strictly dependent on dose or order of session with exactly half of participants in each group receiving that musical genre in the first, moderate dose session and half in the second, high dose session.

Figure 3 contains MEQ30 and CEQ data for this participant-selected musical genre session (Session 3). Higher MEQ30 scores were observed in the Western classical (mean=0.70; $\mathrm{SD}=0.26$ ) compared to overtone-based sessions (mean=0.58; $\mathrm{SD}=0.16$ ) in these sessions, although this between-subject difference was not significant, $p=.41$. CEQ data were again similar between the Western classical (mean=0.29; $S D=0.17$ ) and overtone-based (mean=0.26; $\mathrm{SD}=0.15), p=.79$.

\section{Smoking Abstinence}

Figure 4 contains biologically confirmed smoking abstinence determined by a combination of exhaled carbon dioxide, urinary cotinine, and participant self-report. These values are 
separated into groups by the music selected for the final psilocybin session. Half of participants (2/4) who selected Western classical music for their third session were abstinent at the end of treatment (i.e., 8-weeks following the first psilocybin session/target quit date). These participants remained abstinent through the remainder of assessments (6-month, 12-month, and long-term follow-up). Most (5/6 or $83.3 \%$ ) of the participants who selected overtone-based music were abstinent at the end of treatment. The majority of these participants (4/6 or $66.6 \%$ ) remained abstinent through long-term follow-up.

\section{Discussion}

This analysis used data from an open label trial evaluating the effects of psilocybin administration on smoking cessation to test how different musical genres supporting psychedelic therapy may impact subjective and therapeutic outcomes. Subjective effect measures collected during randomized sessions indicated a trend toward greater mystical experiences for those sessions involving overtone-based compared to Western classical music, although this difference was not statistically significant. Challenging experiences did not differ between these two musical genres with no evident pattern at a group or individual level. Assessment of the target clinical behavior of tobacco cigarette use suggested substantive clinical benefit as reported in the parent study. ${ }^{20,21}$ Importantly, similar clinical benefit was observed based on the participant-selected musical genre for the final psilocybin session with a modest increment for overtone-based selections. Taken together, these findings call into question whether Western classical music typically played in psychedelic sessions holds unique benefit.

As noted in the Introduction, Western classical music has long been assumed to be the standard in psychedelic therapy. 4, 6, 17 The present data challenge this notion that Western classical music - or for that matter any specific genre of music - is an intrinsically superior form of music to support psychedelic therapy, at least for all people at all times. Although we found no significant differences between the two musical genres studied here, several trends suggested the overtone-based playlist resulted in somewhat better outcomes and was preferred by a larger 
portion of this small sample of participants. The lack of superiority of the Western classical playlist is even more interesting considering that some of the overtone-based playlist tracks consisted of sounds without traditionally identifiable melody and/or rhythm, and therefore might not be classified as songs or music by some, although this is highly dependent on one's definitions. This suggests that the sounds capable of supporting psychedelic therapy sessions may go beyond the bounds of traditionally defined musical genres. Interestingly, when participants were able to select their preferred musical genre for a final session, mean mystical experience scores were somewhat higher for sessions with Western classical music compared to overtone-based music - although this comparison was limited by its between-subject nature and was not significant or trending toward significance. This finding implies that individualized selection of music or musical genres may hold some promise and is worthy of experimental follow-up.

Limitations should be noted. This analysis relied on a small sample. However, we utilized individual participant data analysis throughout to demonstrate the consistency of described patterns at a person-level. We also did not collect specific information about the subjective musical experience (e.g., musical liking or acceptance) other than a discrete preference measure. Future efforts designed to optimize individualized selection of music for psychedelic therapy would benefit from such data to empirically test and replicate prior work showing a relationship between positive subjective musical effects and positive therapeutic outcomes. ${ }^{18}$ There was some minor overlap in selections ( $25 \%$ of songs) between musical playlists, perhaps limiting the ability to fully differentiate between the two genres. However, this is partly mitigated by the fact that overlap occurred mostly near the beginning (i.e., pre-onset) and end of sessions (i.e., "come down") rather than during peak drug action. Finally, this study was an open-label design, precluding the ability to evaluate how different musical genres may interact with expectancy or placebo effects. Nevertheless, this open-label context likely better models clinical conditions in which future psychedelic therapy would occur and allowed for specific evaluation of the randomized musical genre manipulation. 
This study provides the first contemporary and within-subject experimental manipulation of session set and setting factors in psychedelic research. It is also the first fully randomized test of different musical genres supporting psychedelic therapy. Although the study is limited by a small sample size, and no significant differences were identified between the musical genres, these data nonetheless failed to identify any clear superiority of Western classical music, which has long been considered the standard. These findings support the idea that developing a process for generating patient-specific musical selections rather than providing standardized music may improve therapeutic outcomes. For example, future work could evaluate how patient-selected music impacts therapeutic effects or identify individual factors predictive of response to varying musical genres or musical features other than genre to individualize session selections. More broadly, these findings emphasize the need for the parametric study of psychedelic session components to either provide improved standardized conditions, or to individualize conditions to improve the therapeutic effects of psychedelic therapy across diverse and varied populations.

\section{Methods}

\section{General Overview and Participants}

This analysis used data from an open-label study on psilocybin for smoking cessation. Additional details about the study design are available in prior manuscripts reporting primary outcomes from that study. ${ }^{20,21}$ Briefly, participants completed a 15 -week treatment period. Participants received a manualized cognitive-behavioral smoking cessation intervention based on cognitive-behavioral therapy (CBT), elements of mindfulness training, and guided imagery for smoking cessation that was delivered in four weekly preparatory meetings prior to the first psilocybin session. Participants in this analysis completed three psilocybin sessions occurring in weeks 5, 7, and 13. The first psilocybin session served as the target quit date for cigarette cessation. Participants returned to the laboratory for 10 weeks following this first psilocybin session to provide urine and breath samples for confirming recent smoking status as well as complete other questionnaire data. Participants completed additional follow-ups at 6 and 12 
months post target quit date as well as a long-term follow-up at an average of 30 months post target quit date.

Participants were required to smoke at least 10 cigarettes per day, report a current desire to stop smoking cigarettes, be physically healthy as determined by medical screening, have no family history of psychotic or bipolar disorders, and have no history of substance use disorder other than nicotine in the past five years. The current analysis focused on a subsample of 10 participants who were randomized to different musical genre playlists during psilocybin sessions. Participants were an average of 51 years old (range=37 to 65 ) and predominantly male ( 8 men, 2 women) and White (9 White, 1 Asian). All participants provided informed consent and the study was approved by the Johns Hopkins University School of Medicine Institutional Review Board.

\section{Psilocybin Sessions and Musical Genres}

Participants completed psilocybin sessions following standard safety guidelines. ${ }^{6}$ Psilocybin was administered in an opaque, size 0 gelatin capsules with approximately $180 \mathrm{ml}$ water. The dose of psilocybin received was $20 \mathrm{mg} / 70 \mathrm{~kg}$ in the first session and $30 \mathrm{mg} / 70 \mathrm{~kg}$ in subsequent sessions. ${ }^{19}$ Staff provided non-directive interpersonal support for managing psilocybin effects during the sessions, but did not deliver smoking cessation-specific content. Additional details on sessions are provided in prior manuscripts from the parent study. 20,21

Participants wore an eye mask and listened to a music program through headphones throughout the session. Music was simultaneously played through room speakers, providing a continual musical experience for the minority of session time when headphones were removed (e.g., when visiting the restroom). The music program was either a "Western classical playlist" containing pieces based on Western classical music similar to that typically used in psychedelic therapy or an "overtone-based playlist" containing pieces that emphasized instruments with a particularly strong overtone signature (Tables S1 and S2). ${ }^{22}$ Similar to the previously developed Western classical playlist, the overtone-based playlist was developed iteratively by two authors (MWJ and AGR) through repetitive listening and refinement of overtone selections and track order 
in line with the expected arc of drug effects during sessions. Common tracks $(25 \%$ of tracks in the Western classical playlist were also used in the overtone-based playlist) occurred mostly near the beginning and end of sessions, corresponding to the pre-onset and "come down" periods, respectively. The order of which playlist used in Sessions 1 versus 2 were randomized for each participant and counterbalanced. Music began at the time of psilocybin administration. Each participant provided approximately 60 min of personally selected music before the first session, which was played during all their sessions approximately 4 hours post-psilocybin administration, roughly corresponding to resolution of peak effects. One participant elected to not include personally selected music. The investigator playlist played at all other points during the session. Prior to Session 3, participants were asked to select which playlist they would like to accompany their third and final psilocybin session.

\section{Outcomes}

\section{Subjective Effect Questionnaires.}

Participants completed the MEQ30 at the completion of each psilocybin session. The MEQ30 has previously been shown to be sensitive to mystical-type subjective effects of psilocybin in survey studies of psilocybin use and laboratory studies of psilocybin administration. ${ }^{23-25}$ Participants also completed the CEQ at the completion of each psilocybin session. This 26-item questionnaire is designed to index challenging experiences related to psychedelic administration. $^{26}$

\section{Smoking Abstinence}

Three measures of cigarette smoking were used to determine smoking abstinence 1) timeline follow-back (TLFB), 2) exhaled carbon monoxide (CO), and 3) urinary cotinine level. Participants completed a smoking TLFB assessment at each study meeting to assess selfreported cigarette smoking. The TLFB is a self-report calendar completed retrospectively by participants indicating the number of cigarettes smoked each day. ${ }^{27}$ Breath exhaled $\mathrm{CO}$ was used as an objective and biological measure of smoking over approximately the past 24 hours. ${ }^{28}$ Breath 
CO was measured using a Bedfont Micro III Smokerlyzer (Haddonfield, NJ). Urine cotinine samples were used as an additional biological and objective measure of nicotine exposure and allowed detection of smoking or other nicotine product use over the prior six days. Seven-day point prevalence abstinence was verified through smoking TLFB data and objective and biological verification of urine cotinine levels of $<200 \mathrm{ng} / \mathrm{mL}$ and breath $\mathrm{CO}$ of $\leq 6 \mathrm{ppm} .^{29-31}$

\section{Data Analysis}

Descriptive statistics were used to summarize subjective effect and smoking abstinence data throughout the study phases. Differences in subjective effect questionnaires (MEQ30 and CEQ) were compared between the two randomized musical genre sessions using a paired $t$-test with effect sizes summarized using Cohen's $d_{z}$. An additional mixed effect model was used to control for session dose while accounting for the repeated measurements with a random participant intercept. A between-subjects $t$-test was also used to summarize differences in subjective effect questionnaire data during the Session 3 (i.e., participant selected musical genre session). All analyses were conducted using $R$ Statistical Analysis with two-tailed tests and $\alpha$ of 0.05 . 


\section{Supporting Information:}

- Description of classical and overtone playlists

Disclosures and Conflicts of Interest: MWJ has served as a paid consultant for AWAKN Life Sciences Inc., Beckley Psychedelic Ltd., Entheogen Biomedical Corp., Field Trip Psychedelics Inc., Mind Medicine Inc., Otsuka Pharmaceutical Development \& Commercialization Inc., and Silo Pharma, Inc. JCS and AGR have no competing interests to declare.

Author Contributions: MWJ developed the concept of incorporating musical genre comparison into the study design. MWJ and AGR developed and implemented the genre comparison design. All authors developed the concept for the analysis. AGR and MWJ conducted data collection. JCS conducted data analysis. JCS developed an initial draft with critical revisions from MWJ and AGR. All authors have given approval to the final version of the manuscript.

Funding Sources: The study was funded by the Heffter Research Institute, the Beckley Foundation, and William Harrison. Support for effort by MWJ and AGR was provided by the Johns Hopkins Center for Psychedelics and Consciousness Research which is funded by the Steven and Alexandra Cohen Foundation and the Tim Ferriss, Matt Mullenweg, Craig Nerenberg, and Blake Mycoskie Collaborative. Support for JCS was provided by the National Institute on Drug Abuse Grant T32DA07209.

Acknowledgments: We thank Alexandre Tannous, M.A., and Twig Harper for recommending selections for the overtone-based playlist. We thank William A. Richards, Ph.D., and Brian D. Richards, Psy.D., for their role in developing the Western classical playlist which had been used in previous studies. 


\section{References}

[1] Johnson, M. W., Hendricks, P. S., Barrett, F. S., and Griffiths, R. R. (2019) Classic psychedelics: an integrative review of epidemiology, therapeutics, mystical experience, and brain network function. Pharmacol. Ther. 197, 83-102.

[2] Nichols, D. E. (2016) Psychedelics. Pharmacol. Rev. 68, 264-355.

[3] Pollan, M. (2019) How to change your mind: What the new science of psychedelics teaches us about consciousness, dying, addiction, depression, and transcendence. Penguin Books, London.

[4] Eisner, B. (1997) Set, setting, and matrix, J. Psychoact. Drugs 29, 213-216.

[5] Hartogsohn, I. (2016) Set and setting, psychedelics and the placebo response: an extrapharmacological perspective on psychopharmacology. J. Psychopharmacol. 30, 12591267.

[6] Johnson, M., Richards, W., and Griffiths, R. (2008) Human hallucinogen research: guidelines for safety. J. Psychopharmacol. 22, 603-620.

[7] Studerus, E., Gamma, A., Kometer, M., and Vollenweider, F. X. (2012) Prediction of psilocybin response in healthy volunteers. PLOS One 7, e30800.

[8] Garcia-Romeu, A., and Richards, W. A. (2018) Current perspectives on psychedelic therapy: use of serotonergic hallucinogens in clinical interventions. Int. Rev. Psychiatry 30, 291316.

[9] Leary, T., Litwin, G. H., and Metzner, R. (1963) Reactions to psilocybin administered in a supportive environment. J. Nerv. Ment. 137, 561-573.

[10] Ludwig, A., Levine, J., Stark, L., and Lazar, R. (1969) A clinical study of LSD treatment in alcoholism, Am. J. Psychiatry 126, 59-69.

[11] Griffiths, R. R., Johnson, M. W., Richards, W. A., Richards, B. D., Jesse, R., MacLean, K. A., Barrett, F. S., Cosimano, M. P., and Klinedinst, M. A. (2018) Psilocybin-occasioned mystical-type experience in combination with meditation and other spiritual practices 
produces enduring positive changes in psychological functioning and in trait measures of prosocial attitudes and behaviors. J. Psychopharmacol. 32, 49-69.

[12] Gaston, E. T., and Eagle Jr, C. T. (1970) The function of music in LSD therapy for alcoholic patients. J. Music Ther. 7, 3-19.

[13] Barrett, F. S., Preller, K. H., and Kaelen, M. (2018) Psychedelics and music: neuroscience and therapeutic implications. Int. Rev. Psychiatry 30, 350-362.

[14] Luo, B., Hu, L., Liu, C., Guo, Y., and Wang, H. (2016) Activation of 5-HT2A/C receptor reduces glycine receptor-mediated currents in cultured auditory cortical neurons. Amino Acids 48, 349-356.

[15] Tang, Z. Q., and Trussell, L. O. (2015) Serotonergic regulation of excitability of principal cells of the dorsal cochlear nucleus. J. Neurosci. 35, 4540-4551.

[16] Barrett, F. S., Preller, K. H., Herdener, M., Janata, P., and Vollenweider, F. X. (2018) Serotonin $2 \mathrm{~A}$ receptor signaling underlies LSD-induced alteration of the neural response to dynamic changes in music. Cereb. Cortex 28, 3939-3950.

[17] Bonny, H. L., and Pahnke, W. N. (1972) The use of music in psychedelic (LSD) psychotherapy. J. Music Ther. 9, 64-87

[18] Kaelen, M., Giribaldi, B., Raine, J., Evans, L., Timmerman, C., Rodriguez, N., Roseman, L., Feilding, A., Nutt, D., and Carhart-Harris, R. (2018) The hidden therapist: evidence for a central role of music in psychedelic therapy. Psychopharmacol. 235, 505-519.

[19] One participant received $20 \mathrm{mg} / 70 \mathrm{~kg}$ psilocybin in both the first and second sessions due to participant concern about potential adverse reactions to higher doses. This option was part of the protocol although the default pattern was to administer the $30 \mathrm{mg} / 70 \mathrm{~kg}$ dose in both the second and third sessions. That participant received $30 \mathrm{mg} / 70 \mathrm{~kg}$ in the third session. 
[20] Johnson, M. W., Garcia-Romeu, A., Cosimano, M. P., and Griffiths, R. R. (2014) Pilot study of the 5-HT2AR agonist psilocybin in the treatment of tobacco addiction. $J$. Psychopharmacol. 28, 983-992.

[21] Johnson, M. W., Garcia-Romeu, A., and Griffiths, R. R. (2017) Long-term follow-up of psilocybin-facilitated smoking cessation. Am. J. Drug Alcohol Abuse 43, 55-60.

[22] Playlists are also available at https://tinyurl.com/yyuuwtth (Classical) and https://tinyurl.com/yy295ufa (Overtone-Based). After the first 3 participants we removed three tracks from the overtone-based playlist (Terry Riley, "A Rainbow in Curved Air", $A$ Rainbow in Curved Air, CBS Records; Steve Reich, "Pulses II", Music for 18 Musicians, ECM Records; Charles B. Kim, "Elegy 1: Isolation", Polis, GenevaMars Music) and made minor adjustments to track order. The playlist in Table S2 is the final version and was used with the majority of participants.

[23] Griffiths, R. R., Johnson, M. W., Carducci, M. A., Umbricht, A., Richards, W. A., Richards, B. D., Cosimano, M. P., and Klinedinst, M. A. (2016) Psilocybin produces substantial and sustained decreases in depression and anxiety in patients with life-threatening cancer: a randomized double-blind trial. J. Psychopharmacol. 30, 1181-1197.

[24] Barrett, F. S., Johnson, M. W., and Griffiths, R. R. (2015) Validation of the revised Mystical Experience Questionnaire in experimental sessions with psilocybin. J. Psychopharmacol. 29, $1182-1190$.

[25] Maclean, K. A., Leoutsakos, J. M., Johnson, M. W., and Griffiths, R. R. (2012) Factor analysis of the Mystical Experience Questionnaire: a study of experiences occasioned by the hallucinogen psilocybin. J. Sci. Study Relig. 51, 721-737.

[26] Barrett, F. S., Bradstreet, M. P., Leoutsakos, J. S., Johnson, M. W., and Griffiths, R. R. (2016) The Challenging Experience Questionnaire: characterization of challenging experiences with psilocybin mushrooms. J. Psychopharmacol. 30, 1279-1295. 
[27] Sobell, L. C., and Sobell, M. B. (1992) Timeline follow-back. In Measuring alcohol consumption, pp 41-72, Springer, Berlin.

[28] Benowitz, N., Jacob, P., Ahijevych, K., Jarvis, M., Hall, S., LeHouezec, J., Hansson, A., Lichtenstein, E., Henningfield, J., and Tsoh, J. (2002) SRNT Subcommittee on Biochemical Verification. Biochemical verification of tobacco use and cessation. Nicotine Tob. Res. 4, 149-159.

[29] Bramer, S. L., and Kallungal, B. A. (2003) Clinical considerations in study designs that use cotinine as a biomarker. Biomarkers 8, 187-203.

[30] Javors, M. A., Hatch, J. P., and Lamb, R. J. (2005) Cut-off levels for breath carbon monoxide as a marker for cigarette smoking. Addiction 100, 159-167.

[31] Middleton, E. T., and Morice, A. H. (2000) Breath carbon monoxide as an indication of smoking habit. Chest 117, 758-763. 


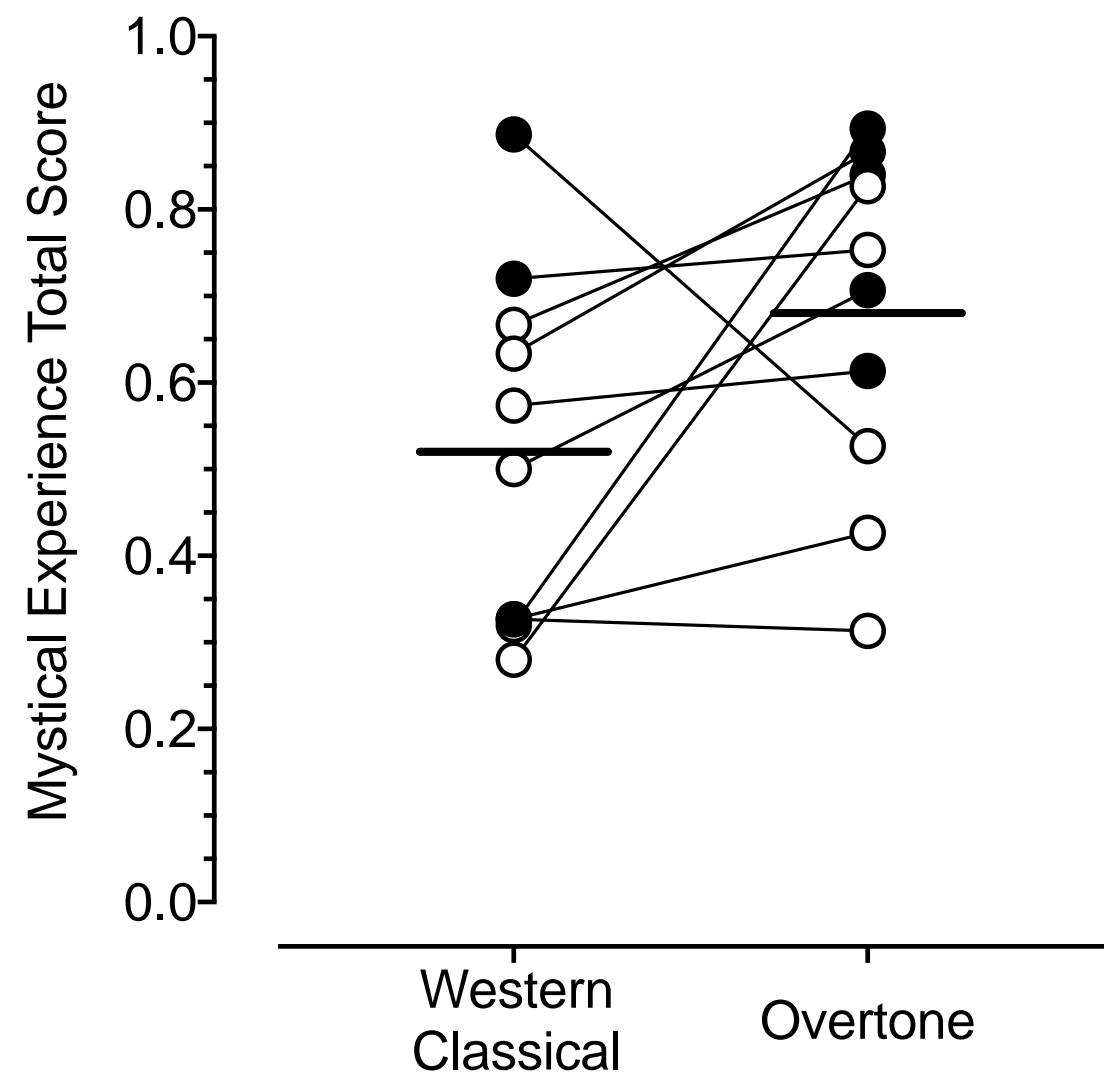

Figure 1. Mystical Experience Questionnaire (MEQ30) Scores by Randomized Musical Genre. Plotted are individual participant data for MEQ30 total score in Sessions 1 and 2. Data points are connected for individual participants. Open symbols are sessions with $20 \mathrm{mg} / 70 \mathrm{~kg}$ psilocybin and filled symbols are sessions with $30 \mathrm{mg} / 70 \mathrm{~kg}$ psilocybin. Thick horizontal lines are group means. 


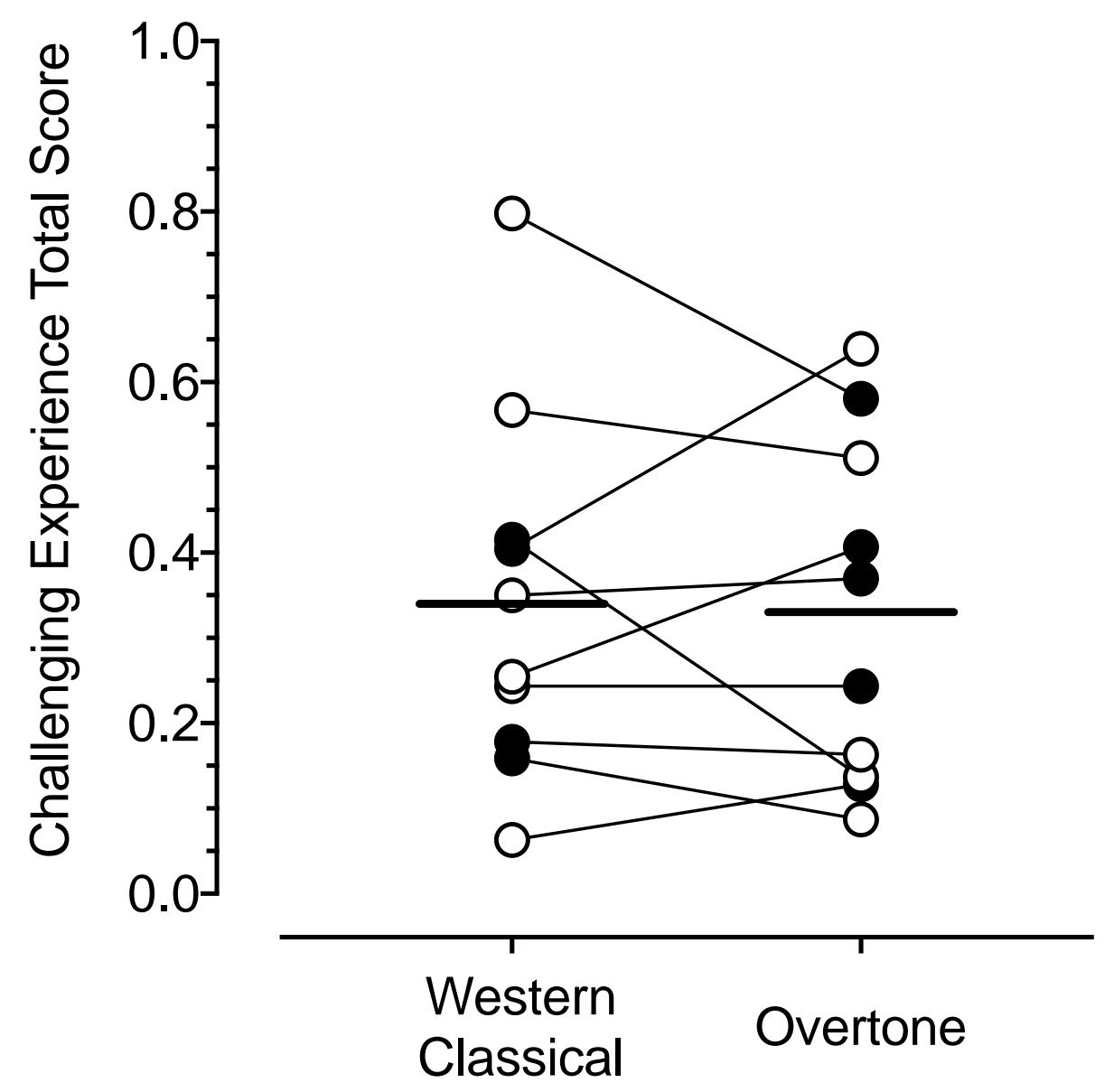

Figure 2. Challenging Experience Questionnaire (CEQ) Scores by Randomized Musical Genre. Plotted are individual participant data for CEQ total score in Sessions 1 and 2. Data points are connected for individual participants. Open symbols are sessions with $20 \mathrm{mg} / 70 \mathrm{~kg}$ psilocybin and filled symbols are sessions with $30 \mathrm{mg} / 70 \mathrm{~kg}$ psilocybin. Thick horizontal lines are group means. 

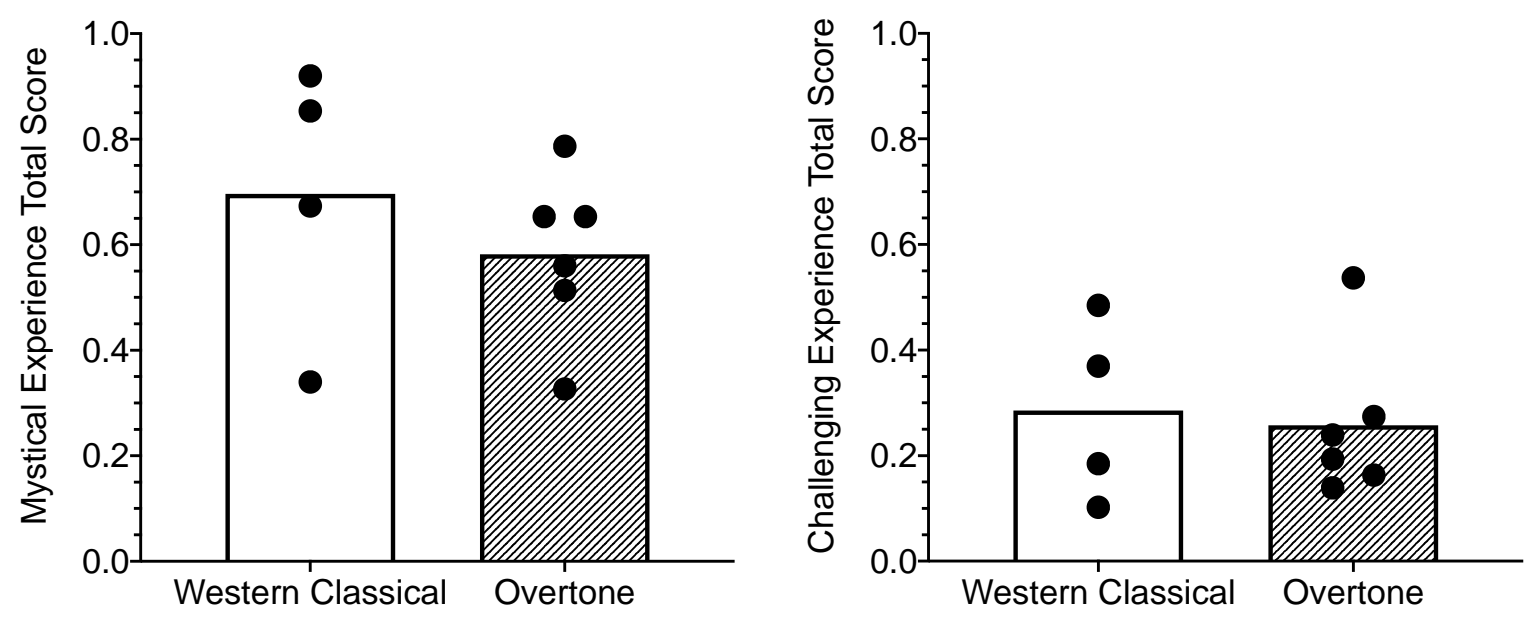

Figure 3. Mystical and Challenging Experience Questionnaire Scores by ParticipantSelected Musical Genre (Session 3). Plotted are individual participant data (black dots) and mean scores for MEQ30 and CEQ total scores in Session 3 (i.e., sessions in which participants could select the musical playlist; see Musical Genre Selection section in the Results). In all cases for Session 3, $30 \mathrm{mg} / 70 \mathrm{~kg}$ of psilocybin was administered. Participants choosing the Western classical playlist are plotted in the open bar, and participants choosing the overtone playlist are plotted in the diagonally striped bar. 


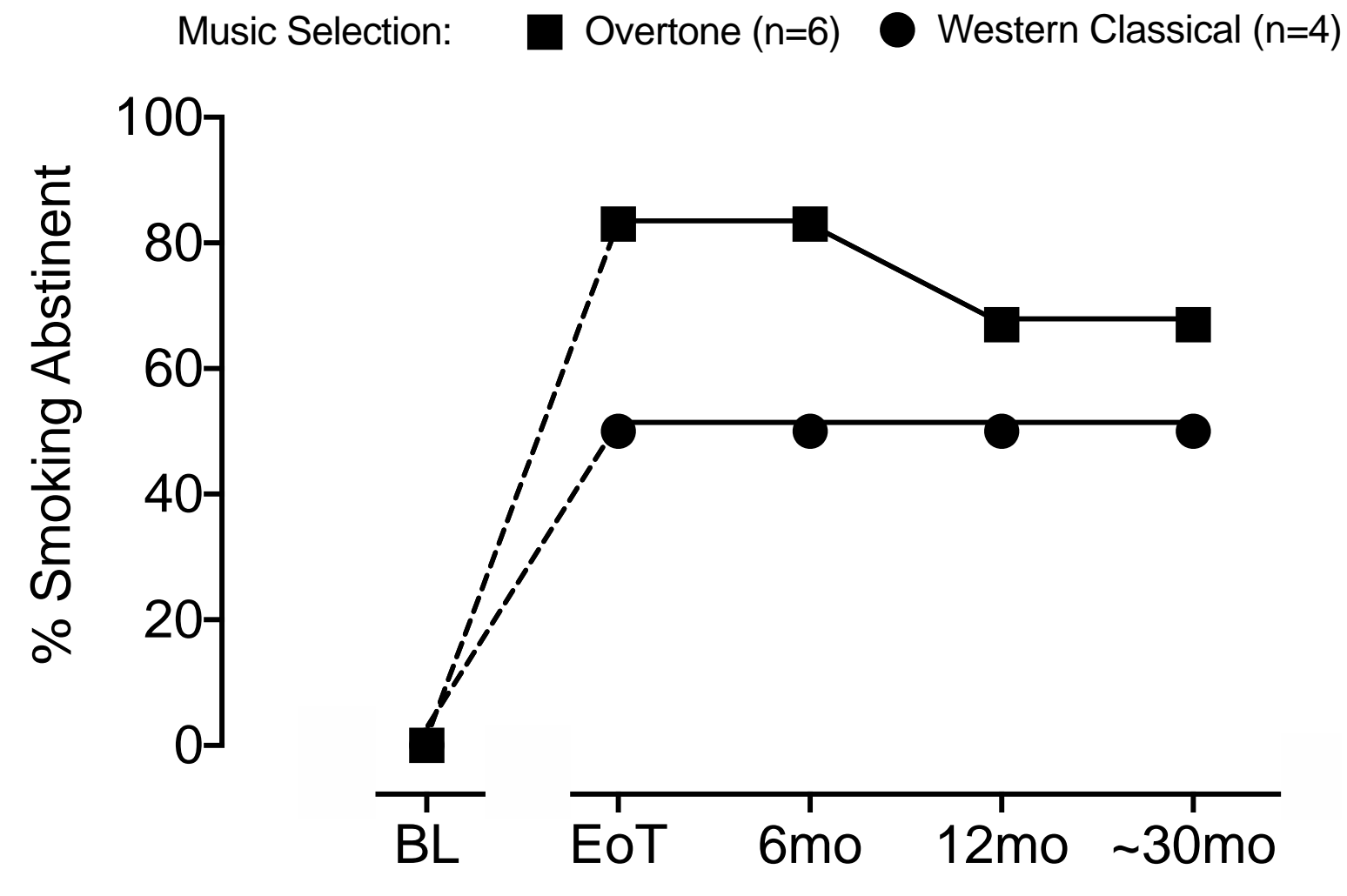

Figure 4. Smoking Abstinence by Musical Genre Selection. Plotted are the percentage of participants with biologically confirmed smoking abstinence during each study phase. $\mathrm{BL}=$ baseline; EoT=end of treatment. Values are presented separately for participants selecting overtone-based playlists (squares) and Western classical playlists (circles) for the third psilocybin session. All participants had sessions with both genre playlists in their first two sessions. 


\section{For Table of Contents Use Only}

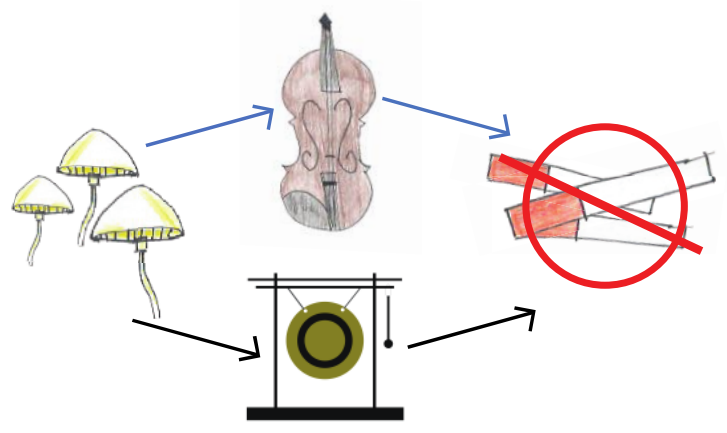

Manuscript title: Set and Setting: A Randomized Study of Different Musical Genres in Supporting Psychedelic Therapy

Names of authors: Justin C. Strickland, Albert Garcia-Romeu, and Matthew W. Johnson

Synopsis: This analysis evaluates effects of musical genre played during sessions of a psilocybin study for tobacco smoking cessation. 


\section{SUPPLEMENTAL MATERIAL}

\section{Set and Setting: A Randomized Study of Different Musical Genres in Supporting \\ Psychedelic Therapy}

Justin C. Strickland ${ }^{1}$, Albert Garcia-Romeu ${ }^{1}$, and Matthew W. Johnson ${ }^{\text {* }}$

${ }^{1}$ Department of Psychiatry and Behavioral Sciences, Johns Hopkins University School of Medicine, 5510 Nathan Shock Drive, Baltimore, MD 21224, USA

${ }^{*}$ Correspondence concerning this article should be addressed to Matthew W. Johnson, Department of Psychiatry and Behavioral Sciences, Johns Hopkins University School of Medicine, 5510 Nathan Shock Drive, Baltimore, MD 21224-6823, USA. Email: mwj@jhu.edu 
Table of Contents

S-3 Table S1

S-6 Table S2

S-8 References 
Table S1. Western Classical Playlist ${ }^{1}$

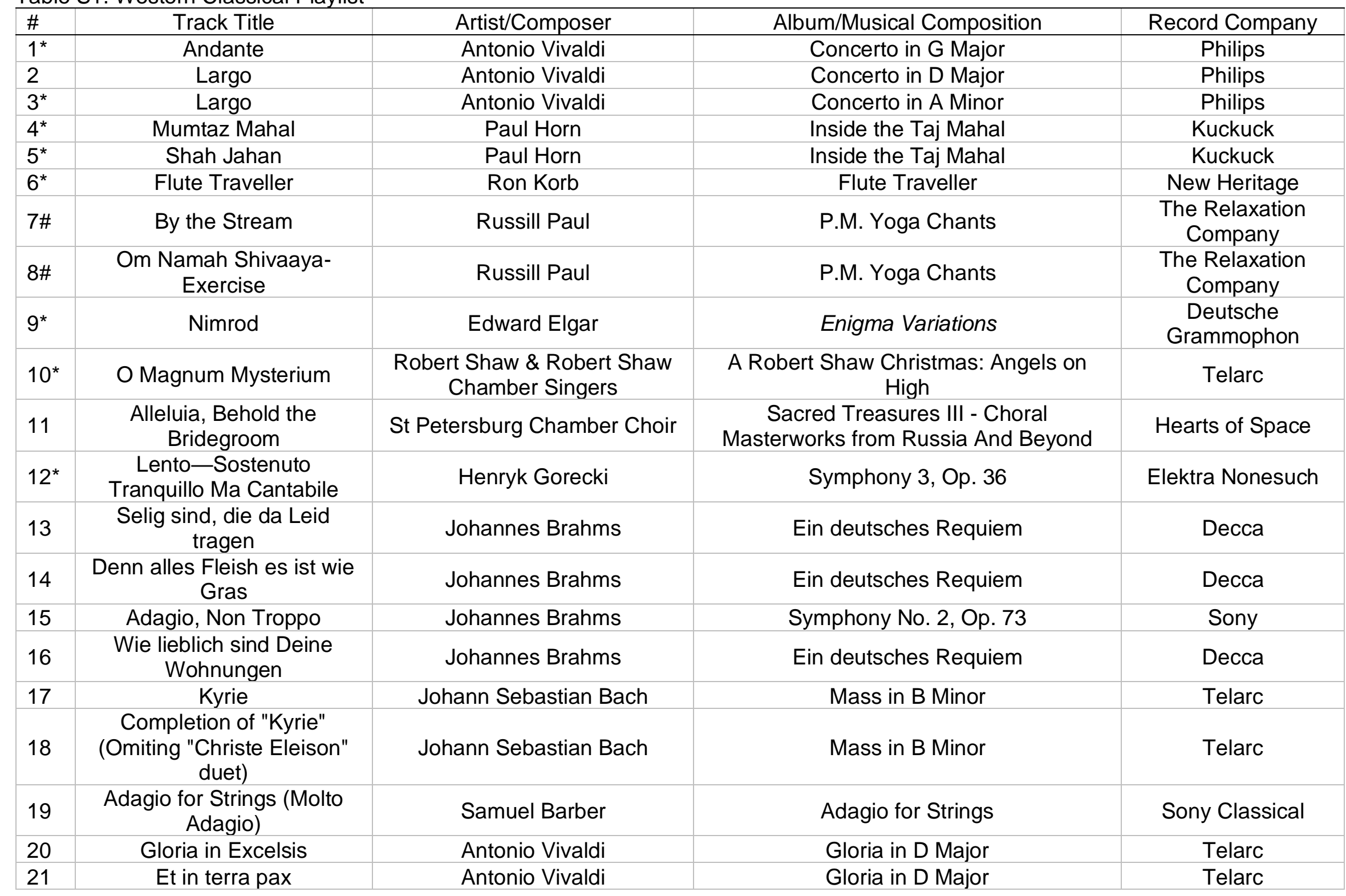




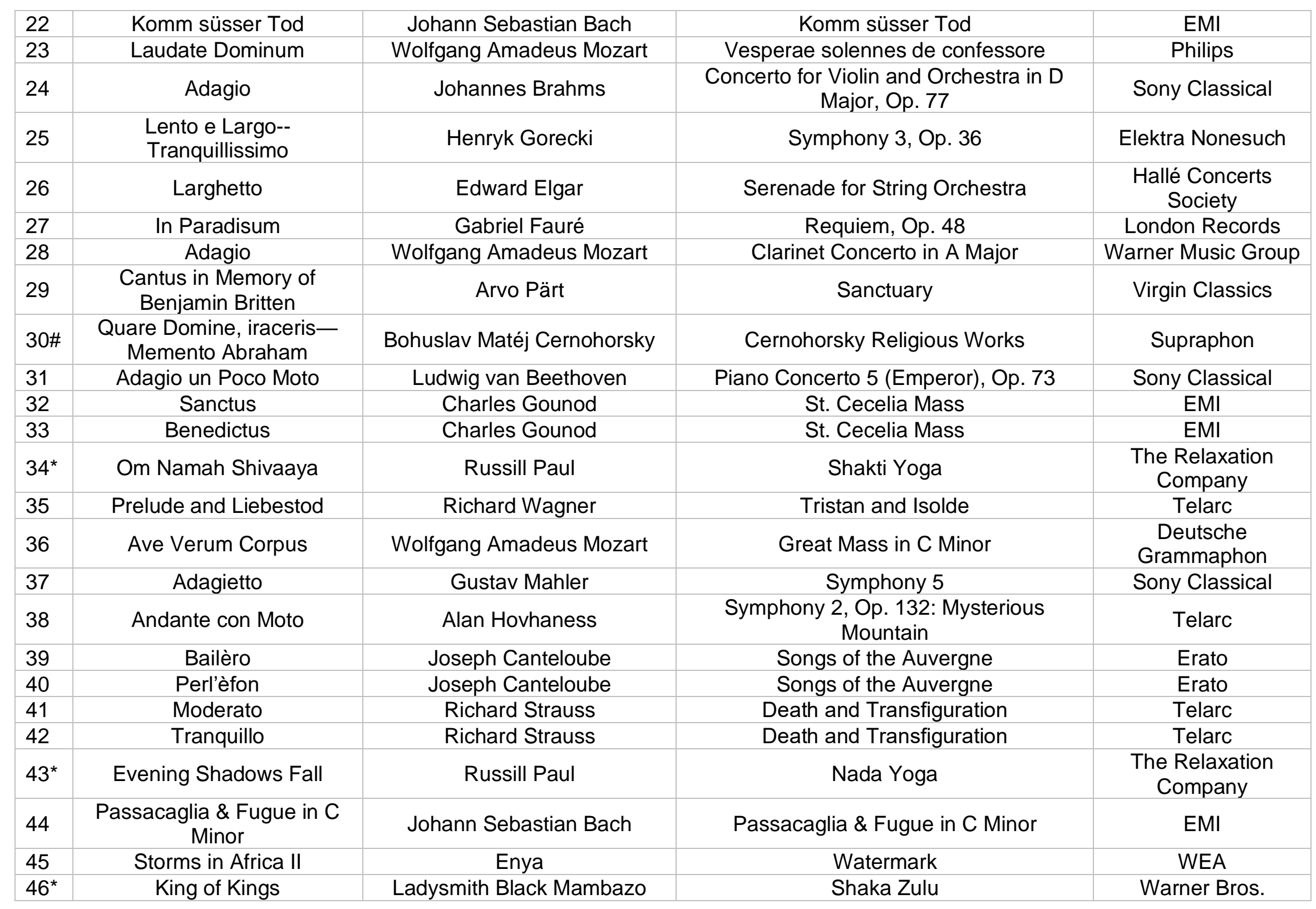




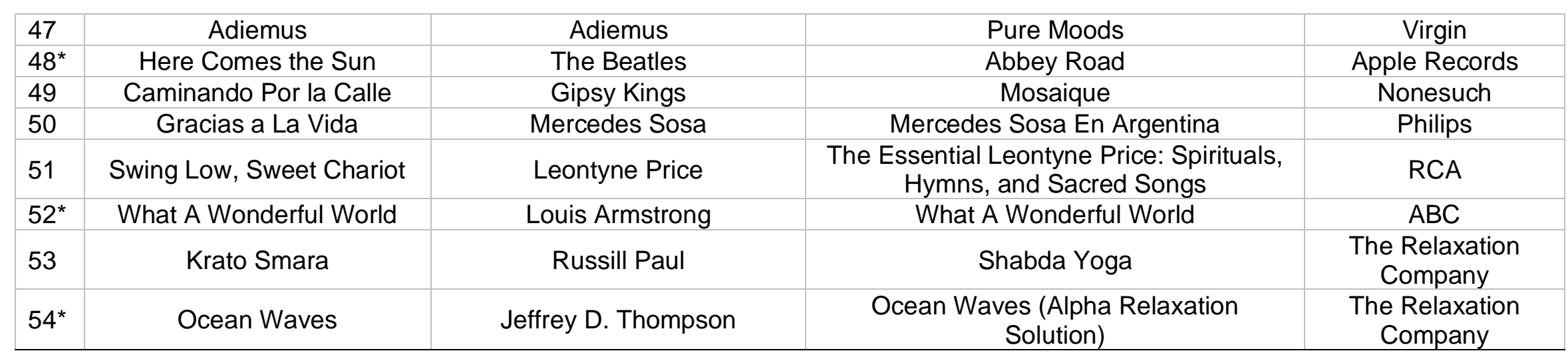

\# songs not available for streaming and not included in playlist link

* songs those shared between playlists 
Table S2. Overtone-Based Playlist ${ }^{2}$

\begin{tabular}{|c|c|c|c|c|}
\hline \# & Track Title & Artist/Composer & Album/Musical Composition & Record Company \\
\hline 1 & Shepherd's Song & Alain Presencer & Singing Bowls of Tibet & Saydisc \\
\hline $2^{*}$ & Andante & Antonio Vivaldi & Concerto in G Major & Andante \\
\hline $3 *$ & Largo & Antonio Vivaldi & Concerto in A Minor & Philips \\
\hline $4^{*}$ & Mumtaz Mahal & Paul Horn & Inside the Taj Mahal & Kuckuck \\
\hline 5 & Earth Chant & $\begin{array}{c}\text { Deborah Van Dyke \& Valerie } \\
\text { Farnsworth }\end{array}$ & $\begin{array}{c}\text { Crystal Voices: The Harmonic Vibrations } \\
\text { of Crystal Singing Bowls }\end{array}$ & Crystal Voices \\
\hline $6 \#$ & Still Water & John August & Meditations: Native American Flute & Lifescapes \\
\hline 7\# & Track 01 & Vitaly Vladimirov & Tibetan Singing Bowls & K-Sound Music \\
\hline $8^{*}$ & Shah Jahan & Paul Horn & Inside the Taj Mahal & Kuckuck \\
\hline $9 *$ & Flute Traveller & Ron Korb & Flute Traveller & New Heritage \\
\hline 10 & Satori (enlightenment) & Tony Scott & Music For Zen Meditation & Verve Records \\
\hline $11^{*}$ & O Magnum Mysterium & $\begin{array}{l}\text { Robert Shaw \& Robert Shaw } \\
\text { Chamber Singers }\end{array}$ & $\begin{array}{c}\text { A Robert Shaw Christmas: Angels on } \\
\text { High }\end{array}$ & Telarc \\
\hline $12^{*}$ & Nimrod & Edward Elgar & Enigma Variations & $\begin{array}{l}\text { Deutsche } \\
\text { Grammophon }\end{array}$ \\
\hline $13^{*}$ & $\begin{array}{c}\text { Lento-Sostenuto } \\
\text { Tranquillo Ma Cantabile } \\
\text { (trimmed version) }\end{array}$ & Henryk Gorecki & Symphony 3, Op. 36 & Elektra Nonesuch \\
\hline 14 & Gravity Waves & $\begin{array}{c}\text { David Hykes \& The Harmonic } \\
\text { Choir }\end{array}$ & Hearing Solar Winds & Harmonic Presence \\
\hline 15\# & Earth & Don Conreaux & Gongs of Our Solar System & $\begin{array}{l}\text { Mysterious } \\
\text { Tremendum }\end{array}$ \\
\hline 16 & $\begin{array}{l}\text { Song from the Balance } \\
\text { Point }\end{array}$ & The Seattle Harmonic Voices & Harmonic Voice & Monktail Records \\
\hline 17 & A Shaman's Spiritwork & Alan Tower & Eternal Presence & $\begin{array}{l}\text { Ancient Future } \\
\text { Music }\end{array}$ \\
\hline 18 & Raga Jogiya Kalingra & Ustad Ali Akbar Khan & Signature Series - Vol. 1 - Three Ragas & AMMP \\
\hline 19 & A Choir of Bells & $\begin{array}{l}\text { Henry Wolff \& Nancy } \\
\text { Hennings }\end{array}$ & Tibetan Bells & BTF Records \\
\hline $20^{*}$ & Om Namah Shivaaya & Russill Paul & Shakti Yoga & $\begin{array}{l}\text { The Relaxation } \\
\text { Company }\end{array}$ \\
\hline 21 & Altitude 27,00 & $\begin{array}{l}\text { Henry Wolff \& Nancy } \\
\text { Hennings }\end{array}$ & Tibetan Bells IV: Bells of Sh'ang Sh'ung & $\begin{array}{l}\text { Henry Wolff \& } \\
\text { Nancy Hennings }\end{array}$ \\
\hline
\end{tabular}




\begin{tabular}{|c|c|c|c|c|}
\hline 22 & Ascending and Descending & $\begin{array}{c}\text { David Hykes \& The Harmonic } \\
\text { Choir }\end{array}$ & Hearing Solar Winds & Harmonic Presence \\
\hline 23 & $\begin{array}{c}\text { Akasha }=\text { Space (Planet- } \\
\text { Suite) }\end{array}$ & Danny Becher & Tibetan Singing Bowls & Oreade Music \\
\hline 24 & A Mystic Meandering & Alan Tower & Eternal Presence & $\begin{array}{l}\text { Ancient Future } \\
\text { Music }\end{array}$ \\
\hline $25 \#$ & $\begin{array}{c}\text { Sound Meditation } 1 \text { (13 } \\
\text { Bowls, } 2 \text { Gongs, Metal Wind } \\
\text { Chimes, Tingsha) }\end{array}$ & Benjamin lobst & Seven Metals - Singing Bowls of Tibet & $\begin{array}{l}\text { Seven Metals } \\
\text { Records }\end{array}$ \\
\hline 26 & $\begin{array}{c}\text { Raga Bhairavi Bhatiyar \& } \\
\text { Raagmala }\end{array}$ & Ustad Ali Akbar Khan & Signature Series - Vol. 2 - Three Ragas & AMMP \\
\hline 27\# & Harmonic Clouds & John Beaulieu & $\begin{array}{c}\text { Calendula: A Suite for Pythagorean } \\
\text { Tuning Forks }\end{array}$ & CD Baby \\
\hline 28 & $\begin{array}{l}285 \mathrm{HZ} \text { - Quantum } \\
\text { Cognition }\end{array}$ & Source Vibrations & Solfeggio Harmonics, Vol. 1 & Source Vibrations \\
\hline 29 & Call of Compassion (Pt. II) & Jonathan Goldman & The Lost Chord & Spirit Music \\
\hline 30\# & Music for Airports $1 / 1$ & Jun Makino & Music for Airports 1/1 & Pinpoint \\
\hline 31\# & Aurora Borealis & Wendy Carlos & Sonic Seasonings & East Side Digital \\
\hline $32^{*}$ & Evening Shadows Fall & Russill Paul & Nada Yoga & $\begin{array}{l}\text { The Relaxation } \\
\text { Company }\end{array}$ \\
\hline 33 & Isis And Osiris & Alice Coltrane & Journey in Satchidananda & Impulse! \\
\hline 34 & Oshusanya & $\begin{array}{c}\text { Masters Of Meian-Ryu, Kimpu- } \\
\text { Ryu, Tozan-Ryu And Kikusi- } \\
\text { Ryu }\end{array}$ & $\begin{array}{c}\text { Japanese Masterpieces for the } \\
\text { Shakuhachi }\end{array}$ & Lyrichord \\
\hline $35^{*}$ & King of Kings & Ladysmith Black Mambazo & Shaka Zulu & Warner Bros. \\
\hline $36^{*}$ & What A Wonderful World & Louis Armstrong & What A Wonderful World & $\mathrm{ABC}$ \\
\hline $37^{*}$ & Here Comes the Sun & The Beatles & Abbey Road & Apple Records \\
\hline $38^{*}$ & Ocean Waves & Jeffrey D. Thompson & $\begin{array}{c}\text { Ocean Waves (Alpha Relaxation } \\
\text { Solution) }\end{array}$ & $\begin{array}{l}\text { The Relaxation } \\
\text { Company }\end{array}$ \\
\hline
\end{tabular}

\# songs not available for streaming and not included in playlist link

* songs those shared between playlists 


\section{References}

[1] Playlist available on Spotify at https://tinyurl.com/yyuuwtth.

[2] Playlist available on Spotify at https://tinyurl.com/yy295ufa. 